\title{
TITLE:
}

\section{Oscillatory links of Fgf signaling and Hes7 in the segmentation clock.}

\author{
$\operatorname{AUTHOR}(\mathrm{S})$ :
}

Harima, Yukiko; Kageyama, Ryoichiro

\section{CITATION:}

Harima, Yukiko ... [et al]. Oscillatory links of Fgf signaling and Hes7 in the segmentation clock.. Current opinion in genetics \& development 2013, 23(4): 484-490

ISSUE DATE:

2013-08

URL:

http://hdl.handle.net/2433/178749

\section{RIGHT:}

(C) 2013 Elsevier Ltd.; This is not the published version. Please cite only the published version.; この論文は出版社版でありません。引用の際に は出版社版をご確認ご利用ください。 


\title{
Oscillatory links of Fgf signaling and Hes7 in the segmentation clock
}

\author{
Yukiko Harima $^{1,2,3}$ and Ryoichiro Kageyama ${ }^{1,3 *}$ \\ ${ }^{1}$ Institute for Virus Research, Kyoto University, Kyoto 606-8507, Japan. \\ ${ }^{2}$ Kyoto University Graduate School of Biostudies, Kyoto 606-8502, Japan. \\ ${ }^{3}$ Japan Science and Technology Agency, CREST, Shogoin-Kawahara, Sakyo-ku, Kyoto \\ 606-8507, Japan
}

*Corresponding author: Ryoichiro Kageyama

Institute for Virus Research

Kyoto University

Shogoin-Kawahara, Sakyo-ku

Kyoto 606-8507

Japan

Tel: $81-75-751-4011$

Fax: 81-75-751-4807

E-mail: rkageyam@virus.kyoto-u.ac.jp 


\section{Summary}

Somitogenesis is controlled by the segmentation clock, where the oscillatory expression of cyclic genes such as Hes7 leads to the periodic expression of Mesp2, a master gene for somite formation. Fgf signaling induces the oscillatory expression of Hes7 while Hes7 drives coupled oscillations in Fgf and Notch signaling, which inhibits and activates Mesp2 expression, respectively. Because of different oscillatory dynamics, oscillation in Fgf signaling dissociates from oscillation in Notch signaling in S-1, a prospective somite region, where Notch signaling induces Mesp2 expression when Fgf signaling becomes off. Thus, oscillation in Fgf signaling regulates the timing of Mesp2 expression and the pace of somitogenesis. In addition, Fgf signaling was found to be a primary target for hypoxia, which causes phenotypic variations of heterozygous mutations in Hes7 or Mesp2, suggesting gene-environment interaction through this signaling.

\section{Introduction}

Somites, metameric structures that later give rise to vertebrae, ribs, skeletal muscles, and subcutaneous tissues, are formed by periodic segmentation of the anterior parts of the presomitic mesoderm (PSM). This periodic event is regulated by a biological clock called the segmentation clock, which involves the oscillatory expression of cyclic genes such as the basic helix-loop-helix (bHLH) gene Hes7 (Figure 1) [1-5]. Genes in the Notch, Fgf, and Wnt pathways are expressed in an oscillatory manner in the mouse PSM [6-9]. One major outcome of such oscillatory expression is periodic activation of the bHLH gene Mesp2, a master gene for somite formation, in the prospective somite region termed $\mathrm{S}-1$ (note that $\mathrm{S} 0$ is the next forming somite while $\mathrm{S}-1$ is the PSM region that will form a somite after S0) (see Figure 3) [10,11]. So, the question is how cyclic genes periodically induce Mesp2 expression in $\mathrm{S}-1$.

Oscillatory expression is halted in the anterior region of the PSM, where overt somite differentiation begins, and the interface between this anterior region and the posterior oscillatory region is called the wavefront. It was previously shown that the wavefront is established by morphogens such as Fgf8 [12,13]. The Fgf8 gene is transcribed only in the posterior end of the PSM, but due to slow degradation, the amount of Fgf8 mRNA gradually decreases in PSM cells, which move anteriorly as the PSM grows posteriorly, forming the posterior to anterior Fgf8 gradient [14]. The 
anterior border of the Fgf gradient corresponds to the wavefront, and after passing this line, PSM cells express Mesp2 because Mesp2 expression is repressed by Fgf8 [11]. Fgf signaling seems to sweep back at a steady speed as the PSM grows, suggesting that the wavefront might regress steadily. However, Mesp2 expression initiates synchronously in the whole S-1 region, and this synchronous expression seems to be important for the subsequent somitogenic processes [11]. These results raise the alternative possibility that the wavefront periodically jumps. Recent studies revealed that oscillators in the Fgf and Notch pathways are essential for periodic induction of Mesp2 expression in S-1.

\section{Fgf signaling induces traveling waves}

The Notch intracellular domain (NICD), an active form of Notch, activates the Hes7 promoter both in in-vitro culture and in-vivo transgenic mouse studies, indicating that Hes7 expression is controlled by Notch signaling [8,15-17]. In agreement with this notion, Hes7 expression is severely down-regulated in the absence of Rbpj, an essential mediator of Notch signaling [8,17]. However, a low level of Hes7 expression still oscillates in the PSM of Rbpj-null mice, suggesting that another signaling may be responsible for oscillatory expression of Hes7 [8,17]. Indeed, Fgf signaling is required for Hes7 expression because Hes7 expression totally disappears in the presence of Fgf inhibitors or in the absence of Fgfrl, an Fgf receptor gene essential for somitogenesis $[8,18,19]$. Thus, Hes7 expression is cooperatively regulated by both Fgf and Notch signaling. In zebrafish, it was previously proposed that non-synchronous oscillatory expression remains in embryos mutant for Notch signaling [20], and it was recently shown that expression of herl, a zebrafish Hes7 homologue, still oscillates nonsynchronously in such mutants [21•]. In contrast, treatment with an inhibitor of Fgf signaling abolished herl expression, whereas transplanted Fgf8-soaked beads induced ectopic traveling waves of herl expression in zebrafish embryos [22••]. These results indicate that Fgf signaling is essential for Hes7/herl oscillations in the PSM.

The stripes of both Hes 7 and herl become narrower as they move anteriorly in the PSM, and this narrowing is owing to slowing oscillations (longer periods) [23,24]. The Fgf8 gradient could be involved in the slowing oscillations because the Fgf level affects the length (or narrowing) of herl stripes [22••]. However, pERK, an effector of Fgf signaling $[13,25]$, is expressed in an oscillatory manner and does not form any apparent gradients in the mouse PSM (see below) [8,26••], indicating that the Fgf8 
gradient is not translated into pERK levels. It was shown that Notch signaling-mediated intercellular coupling regulates the pace of the segmentation clock [27]. Fgf signaling could cross-talk with Notch signaling and thereby control the period of oscillatory expression. Further study will be required to understand the mechanism of how Hes7/her 1 oscillations slow in the anterior PSM and whether Fgf signaling is involved in this slowing.

\section{Coupled and dissociating oscillations of Notch and Fgf signaling}

In the mouse PSM, expression of the bHLH repressor gene Hes 7 oscillates by negative feedback [15]. Hes7 oscillation drives the oscillatory expression of the Notch modulator Lunatic fringe (Lfng), which inhibits Notch signaling [5,17,28-30•]. When Notch signaling is activated by its ligands such as Delta-like1, the transmembrane protein Notch is processed, releasing NICD. Lfng oscillation periodically inhibits Notch signaling, resulting in cyclic formation of NICD in the PSM (Figure 2) [26••,31,32]. It has been shown that NICD activates Mesp2 expression in collaboration with Tbx6, a Tbox protein required for PSM differentiation and segmentation [11,33]. Hes7 also drives the oscillatory expression of Dusp4, a phosphatase of phophorylated ERK (pERK), an effector of Fgf signaling. Owing to Dusp4 oscillation, pERK is periodically dephosphorylated, and the amount of pERK also oscillates in the PSM (Figure 2) $[8,26 \bullet \bullet$. It was shown that Hes7 induces oscillatory expression of another Fgf inhibitor gene, Sprouty4, in the mouse PSM, which could also contribute to pERK oscillation [34]. Thus, Hes7 induces oscillations of both Notch and Fgf signaling in the PSM. Conversely, Notch and Fgf signaling cooperatively up-regulate Hes 7 expression (Figure 2) [7]. These results indicate that Notch and Fgf signaling molecules and Hes7 form the oscillatory gene network. The next question is how the oscillatory expression of NICD, an activator of Mesp2, and pERK, a repressor of Mesp2, induces Mesp2 expression in S-1.

Detailed expression analysis showed that the dynamics is different between NICD and pERK oscillations: NICD expression travels like a wave, narrowing down to a near somite size, while pERK exhibits an On-Off pattern (Figure 3). Narrowing of the NICD expression domain is due to slower oscillation in the anterior PSM compared to the posterior PSM, whereas the pERK oscillation does not slow. When NICD is expressed in the posterior to middle PSM, pERK is also present, inhibiting NICD from 
inducing Mesp2 expression (phases II and III, Figure 3). However, when the NICD expression domain moves and narrows down to the region around $\mathrm{S}-1$, pERK suddenly disappears, which allows NICD to induce Mesp2 expression synchronously in the whole S-1 region (Figure 3, panel furthest to the right) [26••]. Thus, two types of oscillations in Notch and Fgf signaling, which are coupled by Hes7 in the posterior PSM, dissociate in the anterior PSM, and this dissociation allows a group of cells $\left(\mathrm{NICD}^{+} \mathrm{pERK}^{-}\right)$to express Mesp2 simultaneously in the whole S-1 region (Figure 3).

Retinoic acid (RA) signaling forms an opposite gradient to and antagonizes the Fgf8 signaling [35], and it has been proposed that the bistability between antagonistic Fgf and RA gradients leads to jumps of the wavefront [36]. However, this notion remains to be experimentally analyzed. A recent study with the monolayer PSM culture system shows that the phase of Lfng oscillation is delayed in the anterior PSM compared to the posterior PSM [37•]. Interestingly, the phase difference between anterior and posterior cells (phase gradient) was found to be inversely proportional to the sizes of formed segments, suggesting that the segment size or the wavefront jump is predetermined by the phase gradient [37•]. Strikingly, the phase gradient in monolayer PSM culture tissues is maintained even in the absence of an anterior opposing gradient [37•], suggesting that the segment size is controlled independently of an anterior opposing gradient.

\section{Oscillation in Fgf signaling is important for the timing of somite formation}

It was previously thought that Notch signaling function as a pacemaker of the segmentation, while the distance that the wavefront (the anterior boarder of Fgf signaling) travels during one oscillation cycle defines the somite size [1]. However, recent data on the oscillator networks needs revision of this view. Cyclic downregulation of pERK seems to be important for the $\mathrm{S}-1$ cells to express Mesp2 periodically, suggesting that pERK oscillation in Fgf signaling regulates the pace of segmentation (Figures 3 and 4A) [26••]. In contrast, the NICD expression domain narrows in the anterior PSM, and this narrowing is important for the size of somites, because the $\mathrm{NICD}^{+}$region that co-expresses Tbx6 induces Mesp 2 expression after being freed from Fgf-pERK regulation (Figures 3 and 4A) [26・•,31]. Thus, NICD oscillation plays an important role in the spatial regulation of somitogenesis. It was previously shown that increased Fgf activity reduces the somite size without affecting the pace of 
segmentation [12]. Fgf was shown to caudalize the PSM and thereby shift the wavefront anteriorly [12]. In addition, as discussed above, Fgf signaling could be involved in narrowing of Hes7 stripes, which would lead to narrowing of NICD stripes via Lfng. These effects could secondarily affect the somite size. Further analysis will be required to determine the role of Fgf signaling in somite formation to differentiate between direct and indirect activities.

In Hes $7 \mathrm{KO}$ mice, although segmentation is severely defective, Mesp2 is expressed in the $S-1$ region, as observed in wild-type mice $[5,26 \bullet \bullet]$. In the absence of Hes7, Lfng and Dusp4 expression becomes non-oscillatory, and therefore NICD and pERK expression become steady (Figure 4B) [26••]. In Hes7 KO mice, NICD expression continues longer than $\mathrm{pERK}$, forming the $\mathrm{NICD}^{+} \mathrm{pERK}^{-}$region around $\mathrm{S}-1$ where Mesp2 expression is induced. However, time-lapse imaging analysis showed that this Mesp2 expression domain moves steadily as the PSM grows (Figure 4B). As a result, in the absence of Hes7, the onset of Mesp2 expression does not occur simultaneously in the whole $S-1$ region but gradually proceeds from the anterior to posterior even in the same S-1 region (Figure 4B), which may cause the segmentation defects. Thus, although a snapshot of Mesp2 expression is not significantly different between the wild-type and Hes $7 \mathrm{KO}$ embryos (green signals in the left panels of Figure 4A,B), the spatiotemporal profiles of Mesp2 are totally different (green signals in the middle panels of Figure 4A,B) [26••].

The notion that Hes7-induced NICD and pERK oscillations regulate periodic expression of Mesp2 raises the possibility that Hes7 is a key pacemaker of the segmentation clock. It has been suggested that the negative feedback with a delayed timing is essential for sustained oscillation with an appropriate period [38-42], and that negative feedback with shorter delays accelerates the tempo and dampens or abolishes the oscillation. It was recently shown that intronic delays, which include the time required for transcription and splicing of intron sequences, constitute an important part of such proper delays. Due to rapid transcription, splicing events rather than the intron length may be more important for the intronic delay [43]. The Hes7 gene has three introns, and deletion of all three introns reduces the delay by $19 \mathrm{~min}$ and completely abolishes oscillatory expression, leading to steady Hes7 expression and fusion of all somites [44]. In contrast, deletion of two introns of the Hes 7 gene reduces the delay by 5 min and accelerates the pace of the segmentation clock, although the oscillation is 
eventually halted $\left[45^{\bullet}\right]$. Interestingly, these mutant mice have nine cervical vertebrae whereas the wild-type mice have seven, indicating that two more pulses of Hes7 oscillation occur during formation of cervical vertebrae [45•]. It is likely that oscillations in both Notch and Fgf signaling are also accelerated in these mutant mice, suggesting that Hes 7 is a fundamental pacemaker of the segmentation clock.

\section{Hypoxia and Fgf signaling}

Congenital scoliosis, which is caused by vertebral defects, occurs about 1 in 1,000 human live births, and it has been shown that heterozygous mutations in HES7 or MESP2 cause this disease [46••]. Interestingly, some of those who have the same mutations do not have any abnormal vertebrae, indicating partial penetrance. Partial penetrance of vertebral defects is also observed in mice carrying heterozygous mutations in Hes7 or Mesp2 [46••]. Notably, short-term hypoxia induces significantly higher rates and severity of vertebral defects in Hes 7 or Mesp 2 heterozygous mutant mice, suggesting that the phenotypes of genetic disorders are affected by environmental conditions such as hypoxia $[46 \bullet \bullet, 47]$. Hypoxia does not alter $F g f 8$ and $F g f r l$ expression in the PSM, but pERK expression becomes absent or significantly reduced (Figure 2). Furthermore, Hes 7 expression becomes absent or significantly reduced, which is similar to the phenotype caused by treatment with Fgf signaling inhibitors [46••]. These results suggest that hypoxia primarily affects Fgf signaling, although it may also affect other pathways like Wnt and Notch signaling (Figure 2). The mechanism of how hypoxia affects pERK formation remains to be determined.

\section{Perspectives}

In the mouse PSM, NICD makes a traveling wave, while pERK makes an On-Off pattern, and the mechanism by which the dynamics of NICD and pERK oscillations are different remains to be determined. The kinetics of the cell-cell communication may be different between Notch and Fgf signaling. One possibility is that Notch signaling travels relatively slowly because the ligand activates Notch only in adjacent cells, while Fgf is secreted and may rapidly reach distant cells, making the whole population respond simultaneously. Further analysis will be required to determine the parameter values of each signaling communication speed.

Another important aspect of Fgf signaling is its role in elongation of the 
embryonic axis [48-50]. Fgf signaling activates random motility of PSM cells, particularly in the posterior region, but not the proliferation of these cells, and this graded motility (high in the posterior and low in the anterior) seems to contribute to the axis elongation [48]. It remains to be determined how Fgf signaling coordinates the axis elongation with the pace of segmentation.

Recent studies showed that Fgf signaling induces the oscillatory formation of pERK in non-PSM cells such as fibroblasts, and pERK pulses are shown to affect cell cycle progression [51,52]. Thus, the oscillation in Fgf signaling is not specific to the PSM but is involved in other biological events of many cell types. Further analyses will be required to understand the significance and mechanism of oscillation in Fgf signaling activity not only in the segmentation clock but also in other biological events.

\section{References and recommended reading}

1. Pourquié O: Vertebrate segmentation: from cyclic gene networks to scoliosis. Cell 2011, 145:650-663.

2. Oates AC, Morelli LG, Ares S: Patterning embryos with oscillations: structure, function and dynamics of the vertebrate segmentation clock. Development 2012, 139:625-639.

3. Saga Y: The mechanism of somite formation in mice. Curr Opin Genet Dev 2012, 22:331-338.

4. Palmeirim I, Henrique D, Ish-Horowicz D, Pourquié O: Avian hairy gene expression identifies a molecular clock linked to vertebrate segmentation and somitogenesis. Cell 1997, 91:639-648.

5. Bessho Y, Sakata R, Komatsu S, Shiota K, Yamada S, Kageyama R: Dynamic expression and essential functions of Hes7 in somite segmentation. Genes Dev 2001, 15:2642-2647. 
6. Aulehla A, Wehrle C, Brand-Saberi B, Kemler R, Gossler A, Kanzler B, Herrmann BG: Wnt3a plays a major role in the segmentation clock controlling somitogenesis. Dev Cell 2003, 4:395-406.

7. Dequéant M-L, Glynn E, Gaudenz K, Wahl M, Chen J, Mushegian A, Pourquié O: A complex oscillating network of signaling genes underlies the mouse segmentation clock. Science 2006, 314:1595-1598.

8. Niwa Y, Masamizu Y, Liu T, Nakayama R, Deng C-X, Kageyama R: The initiation and propagation of Hes7 oscillation are cooperatively regulated by Fgf and Notch signaling in the somite segmentation clock. Dev Cell 2007, 13:298-304.

9. Krol AJ, Roellig D, Dequéant ML, Tassy O, Glynn E, Hattem G, Mushegian A, Oates AC, Pourquié O: Evolutionary plasticity of segmentation clock networks. Development 2011, 138:2783-2792.

10. Saga Y, Hata N, Koseki H, Taketo MM: Mesp2: a novel mouse gene expressed in the presegmented mesoderm and essential for segmentation initiation. Genes Dev 1997, 11:1827-1839.

11. Oginuma M, Niwa Y, Chapman DL, Saga Y: Mesp2 and Tbx6 cooperatively create periodic patterns coupled with the clock machinery during mouse somitogenesis. Development 2008, 135:2555-2562.

12. Dubrulle J, McGrew MJ, Pourquié O: FGF signaling controls somite boundary position and regulates segmentation clock control of spatiotemporal Hox gene activation. Cell 2001, 106:219-232.

13. Sawada A, Shinya M, Jiang, Y-J, Kawakami A, Kuroiwa A, Takeda H: Fgf/MAPK signalling is a crucial positional cue in somite boundary formation. Development 2001, 128:4873-4880.

14. Dubrulle J, Pourquié O: $f g f 8$ mRNA decay establishes a gradient that couples 
axial elongation to patterning in the vertebrate embryo. Nature 2004, 427:419-422.

15. Bessho Y, Hirata H, Masamizu Y, Kageyama R: Periodic repression by the bHLH factor Hes7 is an essential mechanism for the somite segmentation clock. Genes Dev 2003, 17:1451-1456.

16. Chen J, Kang L, Zhang N: Negative feedback loop formed by Lunatic fringe and Hes7 controls their oscillatory expression during somitogenesis. Genesis 2005, 43:196-204.

17. Ferjentsik Z, Hayashi S, Dale JK, Bessho Y, Herreman A, De Strooper B, del Monte G, de la Pompa JL, Maroto M: Notch is a critical component of the mouse somitogenesis oscillator and is essential for the formation of the somites. PLoS Genet 2009, 5:e1000662.

18. Yamaguchi $\mathrm{T}$, Harpal $\mathrm{K}$, Henkemeyer $\mathrm{M}$, Rossant $\mathrm{J}: \boldsymbol{f g} \boldsymbol{f} \boldsymbol{r} \mathbf{- 1}$ is required for embryonic growth and mesodermal patterning during mouse gastrulation. Genes Dev 1994, 8:3032-3044.

19. Wahl MB, Deng C, Lewandoski M, Pourquié O: FGF signaling acts upstream of the NOTCH and WNT signaling pathways to control segmentation clock oscillations in mouse somitogenesis. Development 2007, 134:4033-4041.

20. Jiang Y-J, Aerne BL, Smithers L, Haddon C, Ish-Horowicz D, Lewis J: Notch signaling and the synchronization of the somite segmentation clock. Nature 2000, 408:475-479.

21 • Delaune EA, François P, Shih NP, Amacher SL: Single-cell-resolution imaging of the impact of Notch signaing and mitosis on segmentation clock dynamics. Dev Cell 2012, 23: 995-1005.

This study showed oscillatory expression of her1 at a single-cell resolution in the zebrafish segmentation clock. It beautifully demonstrated that her1 expression oscillates asynchronously in PSM cells of Notch signaling-mutant zebrafish. Interestingly, her1 
oscillation seems to be linked with mitosis so that cell division makes less noise than previously thought.

22••. Ishimatsu K, Takamatsu A, Takeda $\mathrm{H}$ : Emergence of traveling waves in the zebrafish segmentation clock. Development 2010, 137:1595-1599.

The authors showed that Fgf signaling induces herl oscillation, which forms a traveling wave, in zebrafish embryos. Fgf signaling levels affect the length of herl stripes, indicating that Fgf regulates the period of herl oscillation. The mechanism of how Fgf signaling regulates the period of herl oscillation remains to be determined.

23. Giudicelli F, Ozbudak EM, Wright GJ, Lewis J: Setting the tempo in development: an investigation of the zebrafish somite clock mechanism. PLoS Biol 2007, 5:e150.

24. Masamizu Y, Ohtsuka T, Takashima Y, Nagahara H, Takenaka Y, Yoshikawa K, Okamura H, Kageyama R: Real-time imaging of the somite segmentation clock: revelation of unstable oscillators in the individual presomitic mesoderm cells. Proc Natl Acad Sci USA 2006, 103:1313-1318.

25. Delfini M-C, Dubrulle J, Malapert P, Chal J, Pourquié O: Control of the segmentation process by graded MAPK/ERK activation in the chick embryo. Proc Natl Acad Sci USA 2005, 102:11343-11348.

26••. Niwa Y, Shimojo H, Isomura A, González A, Miyachi H, Kageyama R: Different types of oscillations in Notch and Fgf signaling regulate the spatiotemporal periodicity of somitogenesis. Genes Dev 2011, 25:1115-1120.

This study showed that Hes7 drives coupled oscillations in Fgf and Notch signaling, which inhibits and activates Mesp2 expression, respectively. Oscillation in Fgf signaling dissociates from oscillation in Notch signaling in a prospective somite region, where Notch signaling induces Mesp2 expression in the region where Fgf signaling is off. Thus, oscillation in Fgf signaling regulates the timing of Mesp2 expression and the pace of somitogenesis. 
27. Herrgen L, Ares S, Morelli LG, Schröter C, Jülicher F, Oates AC: Intercellular coupling regulates the period of the segmentation clock. Curr Biol 2010, 20:12441253.

28. Dale JK, Maroto M, Dequeant ML, Malapert P, McGrew M, Pourquié O: Periodic Notch inhibition by Lunatic Fringe underlies the chick segmentation clock. Nature 2003, 421:275-278.

29. Stauber M, Sachidanandan C, Morgenstern C, Ish-Horowicz D: Differential axial requirements for lunatic fringe and Hes7 transcription during mouse somitogenesis. PLoS One 2009, 4:e7996.

30•. Okubo Y, Sugawara T, Abe-Koduka N, Kanno J, Kimura A, Saga Y: Lfng regulates the synchronized oscillation of the mouse segmentation clock via transrepression of Notch signalling. Nat Commun 2012, 3:1141.

This study sought to answer how Lfng regulates synchronized oscillation in the mouse PSM. The authors showed that Lfng represses Notch activity in neighboring cells by modulating Dll1 function, and proposed that this trans-repression is important for synchronized oscillation.

31. Huppert SS, Ilagan MXG, De Strooper B, Kopan R: Analysis of Notch function in presomitic mesoderm suggests a $\gamma$-secretase-independent role for presenilins in somite differentiation. Dev Cell 2005, 8:677-688.

32. Morimoto M, Takahashi Y, Endo M, Saga Y: The Mesp2 transcription factor establishes segmental borders by suppressing Notch activity. Nature 2005, 435:354359.

33. Yasuhiko Y, Haraguchi S, Kitajima S, Takahashi Y, Kanno J, Saga Y: Tbx6mediated Notch signaling controls somite-specific Mesp2 expression. Proc Natl Acad Sci USA 2006, 103:3651-3656.

34. Hayashi S, Shimoda T, Nakajima M, Tsukada Y, Sakumura Y, Dale JK, Maroto M, 
Kohno K, Matsui T, Bessho Y: Sprouty4, an FGF inhibitor, displays cyclic gene expression under the control of the Notch segmentation clock in the mouse PSM. PLoS One 2009, 4:e5603.

35. Diez del Corral R, Olivera-Martinez I, Goriely A, Gale E, Maden M, Storey K: Opposing FGF and retinoid pathways control ventral neural pattern, neuronal differentiation, and segmentation during body axis extension. Neuron 2003, 40:6579 .

36. Goldbeter A, Gonze D, Pourquié O: Sharp developmental thresholds defined through bistability by antagonistic gradients of retinoic acid and FGF signaling. Dev Dyn 2007, 236:1495-1508.

37•. Lauschke VM, Tsiairis CD, François P, Aulehla A: Scaling of embryonic patterning based on phase-gradient encoding. Nature 2013, 493:101-105.

The authors developed a real-time imaging system of Lfng expression in monolayer PSM cultures and showed that the size of formed segments is adjusted (scaled) with that of the remaining PSM tissues. This study also indicated that the segment size is controlled independently of opposing gradients.

38. Lewis J: Autoinhibition with transcriptional delay: a simple mechanism for the zebrafish somitogenesis oscillator. Curr Biol 2003, 13:1398-1408.

39. Monk NAM: Oscillatory expression of Hes1, p53, and NF-kB driven by transcriptional time delays. Curr Biol 2003, 13:1409-1413.

40. Jensen MH, Sneppen K, Tiana G: Sustained oscillations and time delays in gene expression of protein Hes1. FEBS Lett 2003, 541:176-177.

41. Hirata H, Bessho Y, Kokubu H, Masamizu Y, Yamada S, Lewis J, Kageyama R: Instability of Hes7 protein is crucial for the somite segmentation clock. Nat Genet 2004, 36:750-754. 
42. Zeiser S, Rivera O, Kuttler C, Hense B, Lasser R, Winkler G: Oscillations of Hes7 caused by negative autoregulation and ubiquitination. Comput Biol Chem 2008, 32:47-51.

43. Hanisch A, Holder MV, Choorapoikayil S, Gajewski M, Ózbudak EM, Lewis J: The elongation rate of RNA polymerase $I$ in zebrafish and its significance in the somite segmentation clock. Development 2013, 140:444-453,

44. Takashima Y, Ohtsuka T, González A, Miyachi H, Kageyama R: Intronic delay is essential for oscillatory expression in the segmentation clock. Proc Natl Acad Sci USA 2011, 108:3300-3305.

45•. Harima Y, Takashima Y, Ueda Y, Ohtsuka T, Kageyama R: Accelerating the tempo of the segmentation clock by reducing the number of introns in the Hes 7 gene. Cell Rep 2013, 3:in press.

The authors showed that deletion of two introns in the Hes7 gene shortens the transcriptional delay and accelerates the tempo of both Hes 7 oscillation and somite segmentation. This study indicated that Hes 7 is a key regulator of the pace of the segmentation clock.

46••. Sparrow DB, Chapman G, Smith AJ, Mattar MZ, Major JA, O’Reilly VC, Saga Y, Zackai EH, Dormans JP, Alman BA, McGregor L, Kageyama R, Kusumi K, Dunwoodie SL: A mechanism for gene-environment interaction in the etiology of congenital scoliosis. Cell 2012, 149:295-306.

The authors showed that short-term hypoxia induces significantly higher rates and severity of vertebral defects in Hes 7 or Mesp 2 heterozygous mutant mice. It seems that hypoxia primarily affects Fgf signaling. This study indicated that the phenotypes of genetic disorders are affected by environmental conditions such as hypoxia.

47. Dunwoodie SL: The role of hypoxia in development of the mammalian embryo. Dev Cell 2009, 17:755-773.

48. Bénazéraf B, François P, Baker RE, Denans N, Little CD, Pourquié O: A random 
cell motility gradient downstream of FGF controls elongation of an amniote embryo. Nature 2010, 466:248-252.

49. Stulberg MJ, Lin A, Zhao H, Holley SA: Crosstalk between Fgf and Wnt signaling in the zebrafish tailbud. Dev Biol 2012, 369:298-307.

50. Boulet AM, Capecchi MR: Signaling by FGF4 and FGF8 is required for axial elongation of the mouse embryo. Dev Biol 2012, 371:235-245.

51. Nakayama K, Satoh T, Igari A, Kageyama R, Nishida E: FGF induces oscillations of Hes1 expression and Ras/ERK activation. Curr Biol 2008, 18:R332-R334.

52. Albeck JG, Mills GB, Brugge JS: Frequency-modulated pulses of ERK activity transmit quantitative proliferation signals. Mol Cell 2013, 49:1-13.

\section{Figure legends}

Figure 1. Oscillatory expression of Hes7 in the PSM during somite segmentation. Spatiotemporal profiles of Hes7 expression are shown in the lower middle. The $\mathrm{x}$ axis represents time, while the y axis represents space. Anterior is top, and posterior is bottom. The spatial patterns of Hes7 expression at different time points are shown at both sides and on top. Note that the posterior end of the PSM grows posteriorly (downward).

Figure 2. Hes7-mediated coupled oscillations in Fgf and Notch signaling in the segmentation clock. Hes7 drives oscillatory expression of Dusp4, leading to pERK oscillation. Hes7 also drives oscillatory expression of Lfng, leading to oscillatory formation of NICD. Thus, pERK-Dusp4 and Lfng-NICD oscillations are coupled by Hes7 oscillations. Conversely, Hes7 oscillations are cooperatively regulated by Fgf and Notch signaling in the PSM.

Figure 3. Dynamic expression of NICD, pERK and Mesp2 (adapted from [26••]). The 
posterior NICD domain moves anteriorly and narrows, while the pERK domain expands anteriorly, covering the NICD domain (middle two panels). After segmentation, pERK expression is down-regulated, and NICD now induces Mesp2 expression in the S-1 region (panel furthest to the right). Thus, oscillations in Notch signaling periodically segregate a group of synchronized cells, and oscillations in Fgf signaling release these synchronized cells for somitogenesis at the same time.

Figure 4. Spatiotemporal profiles of NICD, pERK and Mesp2 (adapted from [26••]). (A) Spatiotemporal patterns of NICD, pERK and Mesp2 expression in the wild type. Mesp 2 expression is induced periodically by NICD in the whole S-1 region after pERK expression disappears. (B) Spatiotemporal patterns of NICD, pERK and Mesp2 expression in Hes7 $\mathrm{KO}$ mice. In Hes $7 \mathrm{KO}$ mice, pERK expression steadily regresses, and Mesp2 expression also steadily regresses in the anterior PSM after Fgf/ERK signaling is turned off. Thus, Mesp2 expression occurs at different time between the anterior and posterior cells even in the same prospective somites. 


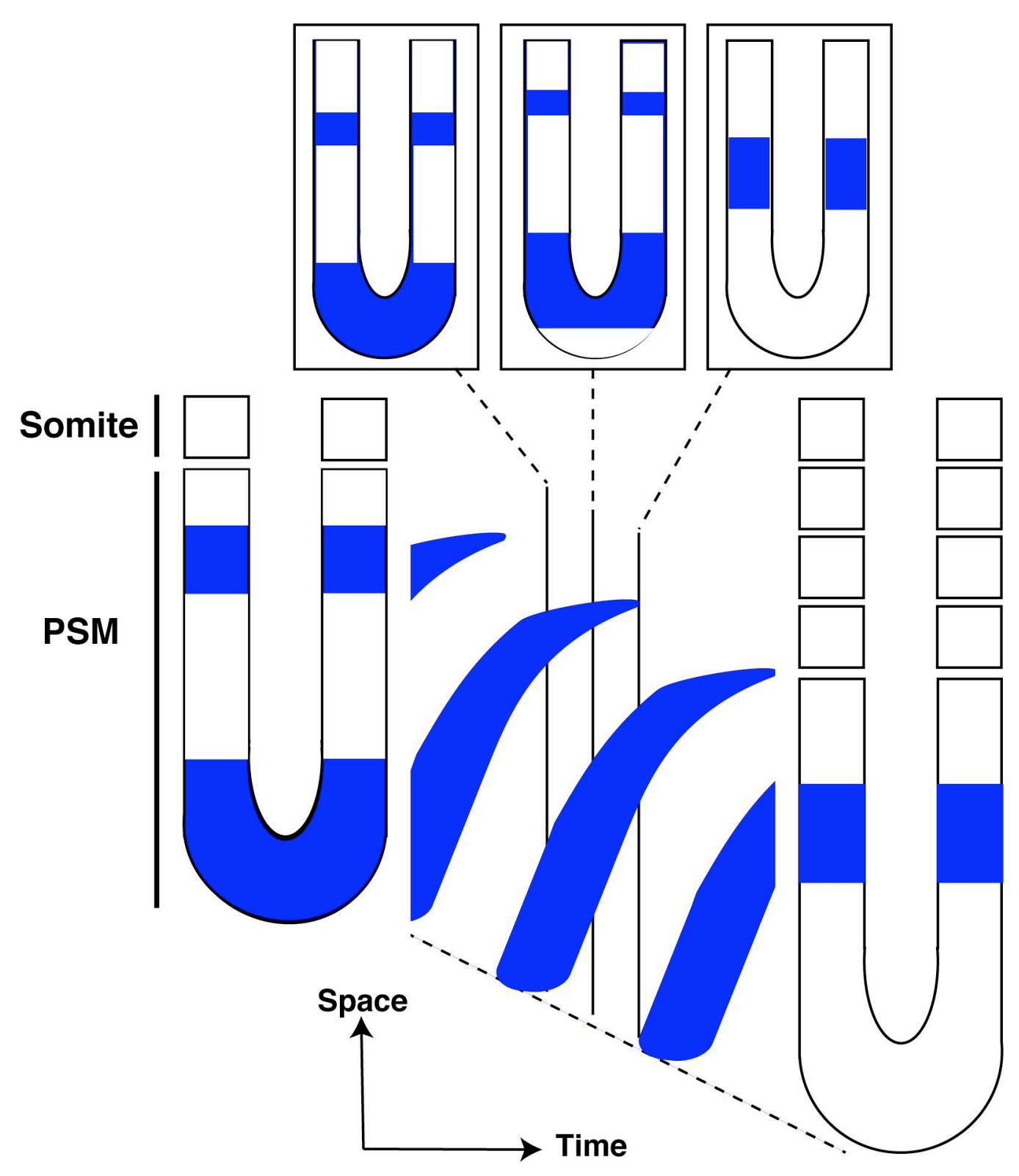

Figure 1 


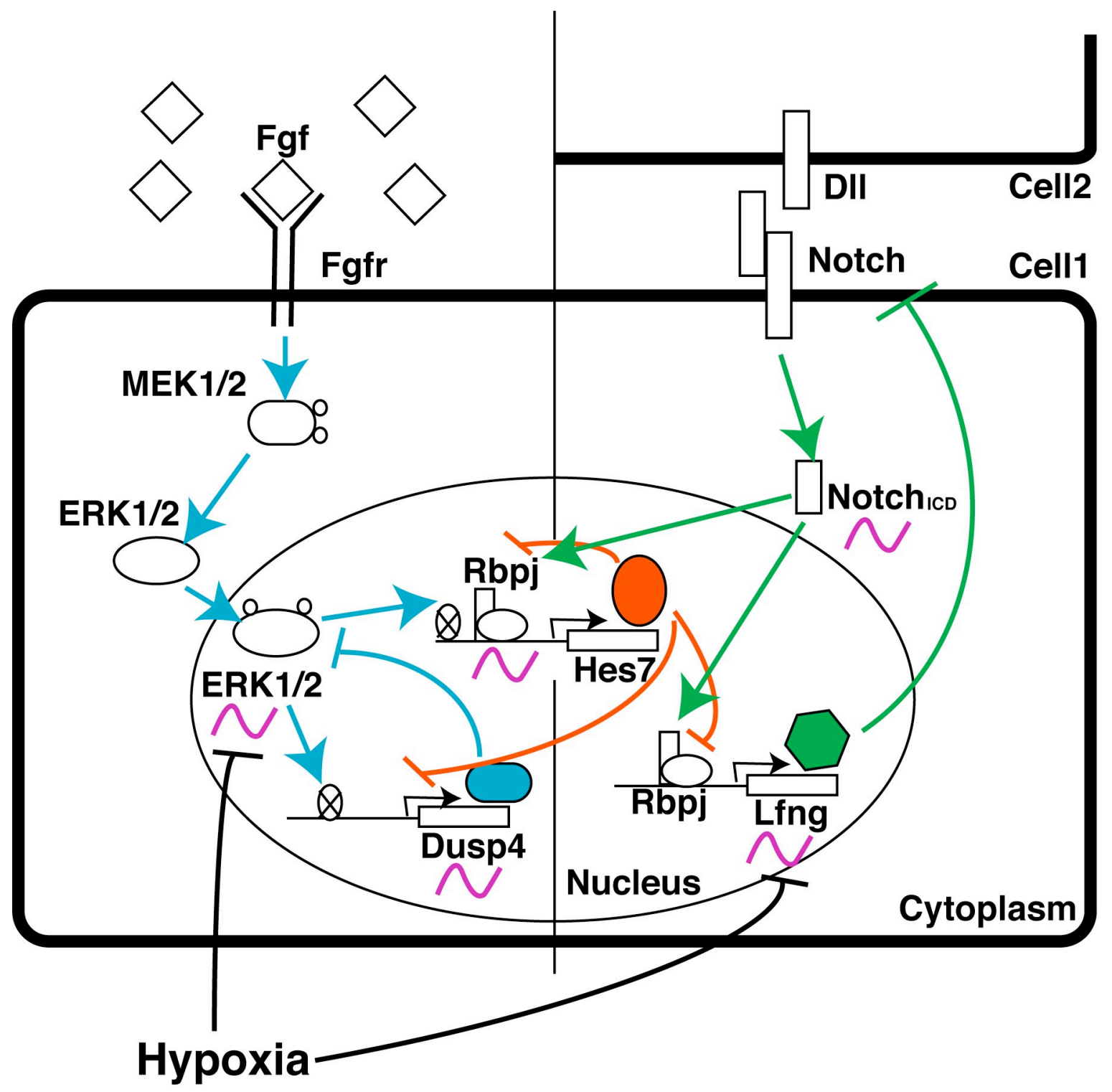

Figure 2 


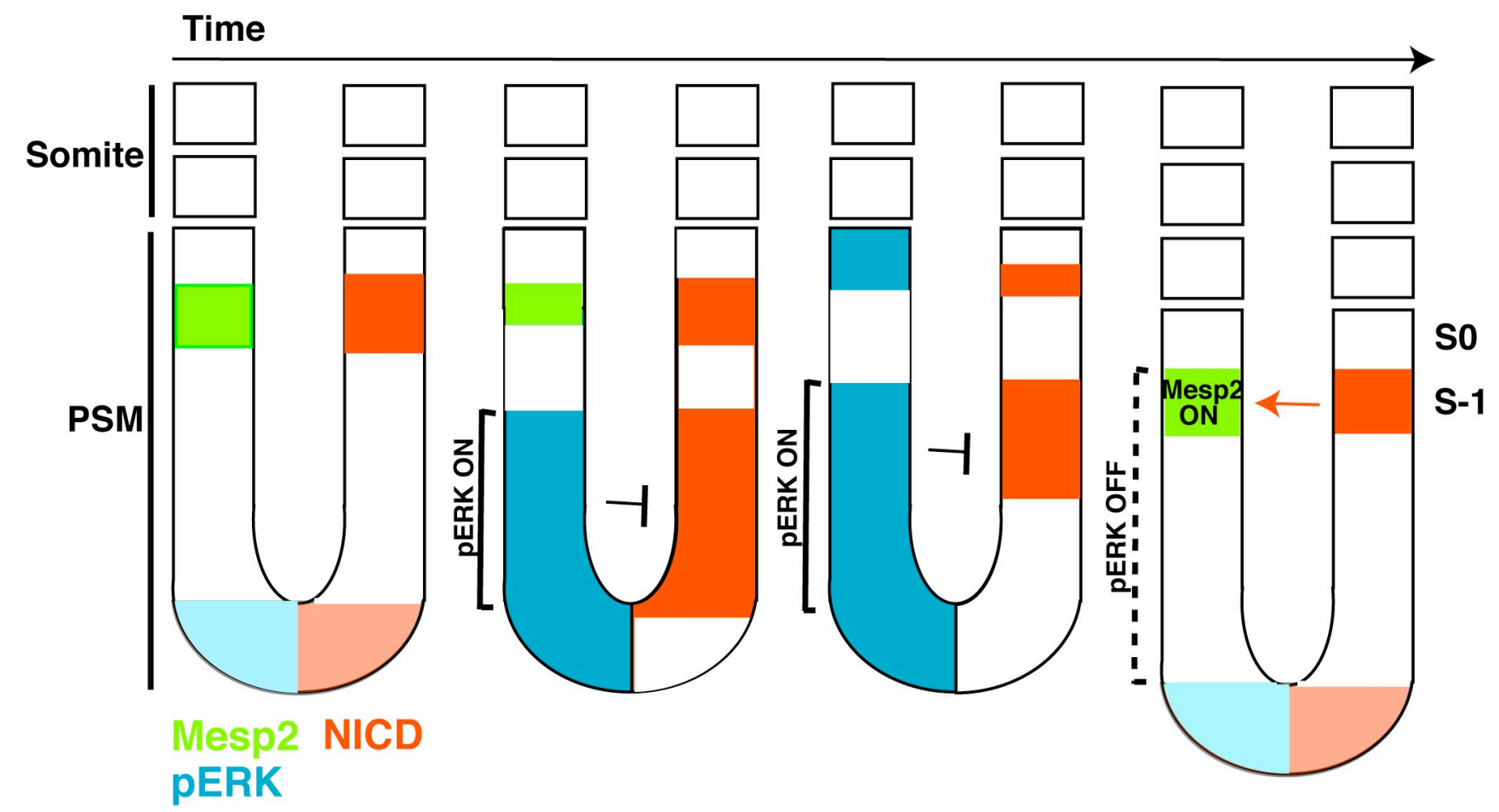

Phase I $\longrightarrow$ Phase II $\longrightarrow$ Phase III Phase I

Figure 3 

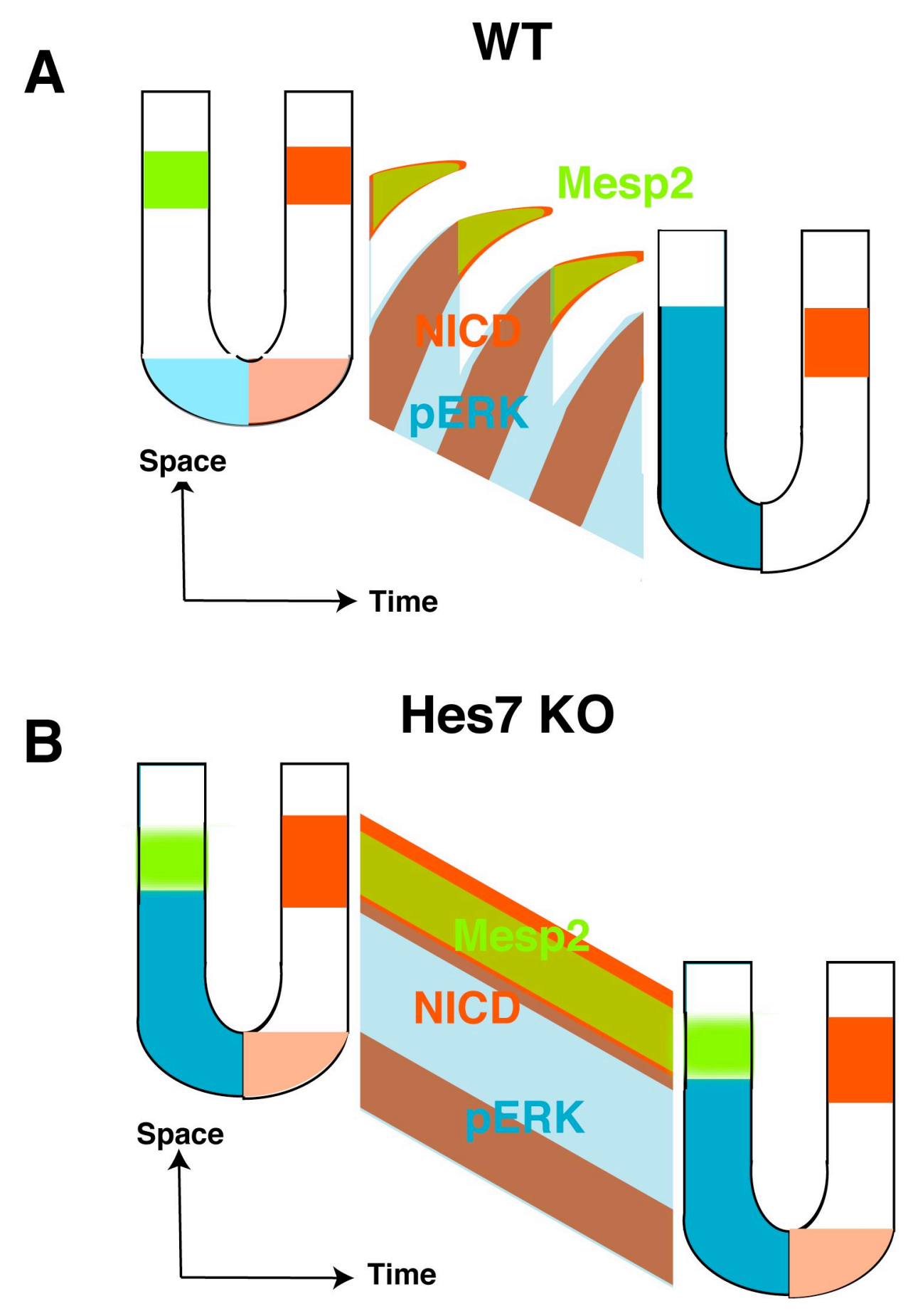

Figure 4 